\title{
EFECTO DE LOS APARATOS ELECTRÓNICOS EN LA SALUD VISUAL DE LOS ALUMNOS DEL NIVEL PRIMARI0 EN LA INSTITUCIÓN EDUCATIVA No 21013 JUAN VALER SANDOVAL
}

\author{
EFFECT OF THE ELECTRONIC DEVICES ON THE VISUAL HEALTH OF THE STUDENTS OF THE \\ PRIMARY LEVEL IN THE EDUCATIONAL INSTITUTION NO. 21013 JUAN VALER SANDOVAL
}

\author{
Mirtha Sussan Trejo López ${ }^{1}$, Miguel Angel Aguilar Luna Victoria ${ }^{1}$, Cristián Iván Escurra Estrada ${ }^{1}$
}

\begin{abstract}
RESUMEN
El objetivo de la investigación fue identificar los efectos de los computadores o equipos móviles en la salud visual de los alumnos del Nivel Primario en la Institución Educativa No 21013 Juan Valer Sandoval. Esta investigación fue de nivel descriptivo, no experimental y transversal. La población estuvo conformada por 45 alumnos de 5to y 6to del nivel primario de la Institución Educativa N 21013 Juan Valer Sandoval del AA.HH. Alberto Fujimori de ambos sexos representando el 100\% del total de la población tomando como criterio de inclusión los alumnos que asistieran a clases el día de la encuesta. Para la recolección de datos previamente se realizó una entrevista con las autoridades y maestros de la institución en el cual se les explicó la finalidad del estudio. Se elaboró el cuestionario conformado por 10 ítems las que fueron respondidas en forma anónima. El examen de agudeza visual se basó en la cartilla de Snellen o tarjeta estandarizada sostenida a una distancia de 20 pies (6 metros) midiendo la claridad o nitidez de la visión. En los resultados se obtuvo que las edades de los niños estuvieron comprendidas de 10 a 13 años, el 67\% tienen conocimiento de los efectos producidos por el uso excesivo de computadores o equipos móviles, el $80 \%$ lo utilizan al día, el 49\% lo emplean menos de una hora, el $42 \%$ presentan malestares, los familiares con problemas a la visión están distribuidos: $39 \%$ padres, $26 \%$ hermanos, $22 \%$ abuelos y $13 \%$ otros familiares. Se llegó a la conclusión de que los efectos de los computadores o equipos móviles en la salud visual está distribuido: $26 \%$ dolor de cabeza, $32 \%$ cansancio en la visión, $16 \%$ visión borrosa, $10 \%$ sensibilidad a la luz y 16\% lagrimeo. El examen de agudeza visual presentó que el $74 \%$ de los alumnos tienen errores de refracción en la visión
\end{abstract}

Palabras clave: Violencia, rendimiento, nivel de evaluación y tipos de violencia.

\section{ABSTRACT}

The objective of the research was to identify the effects of computers or mobile equipment on the visual health of the students of the Primary Level in Educational Institution No. 21013 Juan Valer Sandoval. This investigation was descriptive, not experimental and transversal. The population was conformed by 45 students of 5th and 6th of the primary level of the Educational Institution $N^{\circ} 21013$ Juan Valer Sandoval of the AA.HH. Alberto Fujimori of both sexes, representing $100 \%$ of the total population, taking as an inclusion criterion the students who attended classes on the day of the survey. For the data collection previously an interview was conducted with the authorities and teachers of the institution in which the purpose of the study was explained. The questionnaire consisting of 10 items was prepared and answered anonymously. The visual acuity test was based on the Snellen chart or standardized card held at a distance of 20 feet ( 6 meters) by measuring the clarity or sharpness of the vision. The results showed that the ages of the children were between 10 and 13 years old, $67 \%$ were aware of the effects produced by the excessive use of computers or mobile equipment, $80 \%$ used it daily, $49 \%$ reported it. they use less than one hour, $42 \%$ have discomforts, family members with vision problems are distributed: $39 \%$ parents, $26 \%$ siblings, $22 \%$ grandparents and $13 \%$ other family members. It was concluded that the effects of computers or mobile equipment on visual health is distributed: $26 \%$ headache, $32 \%$ vision fatigue, $16 \%$ blurred vision, $10 \%$ sensitivity to light and $16 \%$ lacrimation. The visual acuity test showed that $74 \%$ of students have refractive errors in vision

Key words: Violence, performance, level of evaluation and types of violence.

1 Universidad Nacional José Faustino Sánchez Carrión (Huacho - Perú). Email: vinvestigación@unjfsc.edu.pe 


\section{INTRODUCCIÓN}

Se estima que el número de niños con discapacidad visual asciende a 19 millones, de los cuales 12 millones la padecen debido a errores de refracción. Aproximadamente 1,4 millones de menores de 15 años sufren ceguera irreversible y necesitan acceso a servicios de rehabilitación visual para optimizar su funcionamiento y reducir la discapacidad (Organización Mundial de la Salud, 2017).

En el mundo existen 39 millones de ciegos (13\% de la población), en Latinoamérica 2,4 millones de ciegos, y en Perú 160,000 ciegos. Para el año 2020 la cifra de ciegos en Latinoamérica alcanzará los 5 millones de habitantes. De acuerdo a diversos estudios, la prevención y el tratamiento oportuno de la pérdida de la visión se cuentan entre las intervenciones de salud más costo-efectivas, en comparación con otros padecimientos. Frente a este panorama, en 1990 la OMS opta por una iniciativa global que une los esfuerzos de las organizaciones no gubernamentales que trabajan en salud visual, agrupadas en la Agencia Internacional para la Prevención de la Ceguera (IAPB) lo que dio nacimiento al Programa VISION 2020: EL DERECHO A LA VISION. En ese mismo año la Federación Internacional de Sociedades Oftalmológicas (IFOS), el Consejo Internacional de Oftalmología (ICO) y la Academia Internacional de Oftalmología (IAO) acuerdan apoyar esta iniciativa. Estas organizaciones promueven el establecimiento de los Comités Nacionales de Prevención de la Ceguera y Programas Nacionales de Salud Ocular a nivel mundial (Ministerio de Salud del Perú, 2014).

VISION 2020 en sus campañas de prevención de la ceguera evitable ha focalizado su atención en cinco problemas oftalmológicos de primer orden: catarata, vicios de refracción y servicios de baja visión, ceguera por enfermedades circunscritas a determinadas zonas geográficas tales como tracoma y oncocercosis, glaucoma y retinopatía diabética (Ministerio de Salud del Perú, 2014).

Los casos de miopía, astigmatismo y otros problemas oculares afectan incluso a niños de 3 años, debido al uso excesivo de aparatos tecnológicos como tablets o smartphones, advirtieron especialistas del ministerio de salud (Flores, 2014).

Si bien la miopía, astigmatismo u otros son genéticos, la exposición por tiempo prolongado a la iluminación de los aparatos electrónicos aumenta la posibilidad de padecerlos con más intensidad. La miopía, el astigmatismo y otros errores refractarios se presentan en niños entre los 6 y 11 años. Sin embargo, ahora, con el daño que producen los gadgets tecnológicos, vemos a menores de 3 años diagnosticados con estos problemas, lo que hace un par de años era muy raro. Los primeros problemas a la visión que se presentan por el uso de las tablets, laptops y similares son sequedad y ardor en los ojos, lagrimeo persistente y dolores de cabeza. Cuando estas situaciones son constantes y tenemos a menores de edad que ya usan lentes, sus medidas y por consiguiente el grosor de las lunas de sus gafas pueden duplicarse en pocos meses. El especialista indicó que en casos extremos, que involucran otros factores como el sedentarismo, el sobrepeso o la diabetes, se deriva en una discapacidad visual que no puede ser corregida con facilidad, e incluso en casos de ceguera. Señaló Harvy Honorio, coordinador de la Estrategia Sanitaria Nacional de Salud Ocular y Prevención de la Ceguera del Minsa (Flores, 2014).

El aumento del número de niños en etapa escolar que usan computadoras, tablets, laptops, celulares, y otros dispositivos electrónicos, ocasionan problemas de visión en el infante. En el Perú se estima que seis de cada 100 niñas y niños de 3 a 11 años tienen trastornos oculares por necesidad de uso $\mathrm{d}$ e $\quad 1$ e $\mathrm{n} \mathrm{t}$ e s A nivel mundial el número de niños menores de 16 años con discapacidad visual asciende a 19 millones, de los cuales 12 millones son por errores de refracción como miopía, hipermetropía, astigmatismo o estrabismo, fácilmente diagnosticables y corregibles, indicó el Instituto Nacional de Salud del Niño (RPP Noticias, 2016).

\section{MATERIALES Y MÉTODOS}

Esta investigación fue de nivel descriptivo, no experimental y transversal. La población estuvo conformada por 45 alumnos de 5to y 6to del nivel primario de la Institución Educativa $N^{\circ} 21013$ Juan Valer Sandoval, de ambos sexos representando el $100 \%$ del total de la población tomando como criterio de inclusión los alumnos que asistieran a clases el día de la encuesta.

Se utilizó la técnica documental, que consistió en recolección de información por medio de bibliografías, revistas, medios impresos, periódicos, artículos, boletines y monografías las cuales se realizó la revisión, ordenamiento y análisis de información bibliográfica.

Se empleó la técnica de la encuesta y se elaboró el 
cuestionario conformado por 10 ítems las que fueron respondidas en forma anónima, las respuestas resultantes fueron codificadas y analizadas con el fin de determinar claridad, coherencia y pertinencia de las mismas a fin de obtener sinceridad en sus declaraciones. Cada uno de los ítems consta de un enunciado y las opciones de respuesta. El examen de agudeza visual se basó en la caretilla de Snellen o tarjeta estandarizada sostenida a una distancia de 20 pies (6 metros) midiendo la claridad o nitidez de la visión. El primer número se refiere a la distancia en pies a la que se encuentra el sujeto de la cartilla. El segundo número indica a la cual una persona con visión normal puede leer la misma línea. La evaluación de la agudeza visual dentro del rango normal se encuentra de 20/20 - 20/30

Se utilizó la estadística descriptiva con la finalidad de identificar apropiadamente las características de la población en estudio mediante tablas y gráficos estadísticos respetando las etapas de la investigación.

\section{RESULTADOS}

Un total de 45 alumnos participaron del estudio respondiendo el cuestionario, sus edades estuvieron comprendidas de 10 a 13 años, el 55.6\% (25 mujeres) y 44.4\% (20 varones), el 67\% (30 alumnos) tienen conocimiento de los efectos producidos por el uso excesivo de computadores o equipos móviles, el $84 \%$ (34 alumnos) cuentan con un computador o equipo móvil, el 80\% (36 alumnos) lo utilizan al día, el 49\% (22 alumnos) lo emplean menos de una hora, el 58\% (26 alumnos) lo usan para investigar temas de sus trabajos, el 22\%(10 alumnos) para socializar, el $18 \%$ ( 8 alumnos) para jugar y el $2 \%$ ( 1 alumno) para tomar apuntes, el 42\% (19 alumnos) presentan malestares durante el uso de computadores o equipos móviles y el 51\% ( 23 alumnos) tienen problemas sus familiares a la visión de los cuales el 20\%(9 alumnos) son los padres, 13\% (6 alumnos) los hermanos, $11 \%$ (5 alumnos) los abuelos y 7\% (3 alumnos) otros familiares.

Tabla 1. Conocimiento sobre los efectos producidos por el uso excesivo de computadores o equipos móviles

\begin{tabular}{ccc}
\hline RESPUESTA & $\mathbf{N}^{0}$ & $\%$ \\
\hline $\mathrm{Si}$ & 30 & 67,0 \\
No & 15 & 33,0 \\
\hline TOTAL & $\mathbf{4 5}$ & $\mathbf{1 0 0 , 0}$ \\
\hline
\end{tabular}

Tabla 2. Cuenta con un computador o equipo móvil

\begin{tabular}{rrr}
\hline RESPUESTA & $\mathbf{N}^{\circ}$ & $\%$ \\
\hline Si & 38 & 84,0 \\
No & 7 & 16,0 \\
\hline TOTAL & $\mathbf{4 5}$ & $\mathbf{1 0 0 , 0}$ \\
\hline
\end{tabular}

Tabla 3. Frecuencia de uso del computador o equipo móvil

\begin{tabular}{lrr}
\hline $\begin{array}{l}\text { FRECUENCIA DEL USO } \\
\text { DEL COMPUTADOR O }\end{array}$ & $\mathbf{N}^{\mathbf{0}}$ & $\%$ \\
$\quad$ EQUIPO MÓVIL & & \\
\hline Al día & 36 & 80,0 \\
A la semana & 7 & 16,0 \\
Cada dos semanas & 1 & 2,0 \\
Cada tres semanas & 1 & 2,0 \\
\hline TOTAL & 45 & 100,0 \\
\hline
\end{tabular}

Tabla 4. Tiempo invertido en uso de computadores o equipos móviles de forma ininterrumpida

\begin{tabular}{lrr}
\hline \multicolumn{1}{c}{ TIEMPO INVERTIDO } & $\mathbf{N}^{\circ}$ & $\%$ \\
\hline Menos de 1 hora & 22 & 49,0 \\
Entre 1 y 2 horas & 10 & 22,0 \\
Entre 2 y 3 horas & 2 & 4,0 \\
Entre 3 y 4 horas & 3 & 7,0 \\
Más de 4 horas & 8 & 18,0 \\
\hline TOTAL & $\mathbf{4 5}$ & $\mathbf{1 0 0 , 0}$ \\
\hline
\end{tabular}

Tabla 5. Uso de los aparatos electrónicos

\begin{tabular}{lrr}
\hline $\begin{array}{c}\text { USO DE LOS } \\
\text { APARATOS } \\
\text { ELECTRONICOS }\end{array}$ & No & $\%$ \\
\hline Socializar & 10 & 22,0 \\
Jugar & 8 & 18,0 \\
Investigar & 26 & 58,0 \\
Tomar apuntes & 1 & 2,0 \\
\hline TOTAL & $\mathbf{4 5}$ & $\mathbf{1 0 0 , 0}$ \\
\hline
\end{tabular}

Tabla 6. Efectos por el uso de computadores o equipos móviles

\begin{tabular}{lrr}
\hline $\begin{array}{l}\text { EFECTOS POR USO DE } \\
\text { COMPUTADORES O }\end{array}$ & No & $\%$ \\
EQUIPOS MÓVILES & & \\
\hline Dolor de cabeza & 5 & 26,0 \\
Cansancio en la visión & 6 & 32,0 \\
Visión borrosa & 3 & 16,0 \\
Sensibilidad a la luz & 2 & 10,0 \\
Lagrimeo & 3 & 16,0 \\
\hline TOTAL & $\mathbf{1 9}$ & $\mathbf{1 0 0 , 0}$ \\
\hline
\end{tabular}


Tabla 7. Parentesco familiar con problemas de visión

\begin{tabular}{lrc}
\hline \multicolumn{1}{c}{$\begin{array}{c}\text { PARENTESCO } \\
\text { FAMILIAR }\end{array}$} & $\mathbf{N}^{\mathbf{0}}$ & $\%$ \\
\hline Padres & 9 & 39,0 \\
Hermanos & 6 & 26,0 \\
Abuelos & 5 & 22,0 \\
Otros familiares & 3 & 13,0 \\
\hline TOTAL & $\mathbf{2 3}$ & $\mathbf{1 0 0 , 0}$ \\
\hline
\end{tabular}

\section{DISCUSIÓN}

El $67 \%$ de los alumnos manifiestan tener conocimiento de los efectos producidos por el uso excesivo de los computadores o equipos móviles y el $71 \%$ invierten no más de 2 horas al uso ininterrumpido resultados diferentes a lo reportado por Acosta, Cordero y Pérez (2017). De los alumnos que presentan malestares indica que los efectos por el uso del computador o equipos móviles el $26 \%$ tienen dolor de cabeza, el 16\% visión borrosa, el $10 \%$ sensibilidad a la luz y el $16 \%$ lagrimeo estos resultados son similares a lo realizado por Acosta, Cordero y Pérez (2017). El 32\% presentan cansancio en la visión estos síntomas son indicios de la fatiga visual, una condición que advierte al organismo que la salud oftálmica está en riesgo.

El uso excesivo de los dispositivos electrónicos podría cansar e irritar los ojos de los niños produciendo sequedad, problemas de ojo seco, ardor en los ojos, lagrimeo persistente, dolor de cabeza, estrés visual. "Cuando estas situaciones son constantes y tenemos a escolares que ya usan lentes, el grosor de las lunas de sus lentes podría duplicarse en pocos meses", explicó la Dra. María Angélica Rivas, Oftalmóloga pediatra del instituto de salud del Niño San Borja (RPP Noticias, 2016).

Uno cuando está en la pantalla, en la computadora o en la tablet, lo que hacemos es dejar de parpadear por estar concentrados. Eso hace que el ojo se seque, lo cual genera todo tipo de molestias en el paciente, incluso le molesta solo la luz. Es solo por sequedad. Si parpadeáramos un poco más seguidos, esto haría que no se evapore las lágrimas y podríamos ver mejor. Lo otro es alimentarse bien, lo que hace mal en cuerpo al general (azúcar y grasa en exceso) le va a hacer mal también a la vista", explicó el doctor Luis Izquierdo Villavicencio que sobre su elección como el mejor oftalmólogo del mundo y el estado de su disciplina en el Perú. (Huerta, 2016).

E1 74\% de los alumnos tienen errores de refracción en la visión y el $25 \%$ tienen una visión normal, por ello como promotores de la salud tenemos la obligación de promover el uso adecuado de los aparatos electrónicos y promover la corrección de la visión con el uso de anteojos. La medida de la agudeza visual es un parámetro que resulta necesario para evaluar el estado de salud ocular, si bien existen enfermedades oculares que pueden afectar mínimamente a la agudeza visual o incluso no hacerlo, otras pueden causar su descenso de forma brusca o aguda. La agudeza visual es necesaria para realizar la refracción, tanto de lejos como de cerca y de esta manera clasificar o determinar grados de incapacidad en el alumno. 


\section{BIBLIOGRAFÍA}

Acosta, M., Cordero, Y. y Pérez, J. (2017). Influencia del Uso Excesivo de la Tecnología en la Salud Visual de los Estudiantes del 1er y 2 do Año de la I.E. "Simón Rodríguez". E1 PALITO, Municipio Marcano Estado Nueva Esparta. República Bolivariana Venezuela.

Flores, D. (2014). MINSA: Uso excesivo de tablets y smartphones causa miopía en niños. Perú 21. Obtenido de https:/peru21.pe/ vida/minsa-excesivo-tabletssmartphones-causa-miopia-niños141719

Huertas, E. (2016). El mejor oftalmólogo del mundo da consejos para cuidar tu vista. RPP Noticias. Obtenido de htpp://vital.rpp.pe/salud/el-mejoroftamologo-del-mundo-daconsejos-para-cuidar-tu-vistanoticia-967125 http://www.repositorioacademico.usmp .edu.pe/bitstream/usmp/2887/3/cor dova.gv.pdf

h t t p : / / w w w . a a o org / s a lud ocular/consejos/que-significa-unavision-2020

http://www.rehueong.com.ar/sities/defa ult/files/agudeza\%20visual.pdf

http://media.axon.es/pdf/80814.pdf

http//www.provisu.ch/images/PDF/Sne llenchart.es.pdf

Ministerio de Salud del Perú (2014). Plan de la estrategia sanitaria nacional de salud ocular y prevención de la ceguera. Lima, $\mathrm{P}$ e $\mathrm{r}$ ú $\quad \mathrm{O} b \mathrm{t}$ e $\mathrm{n}$ i d o d e http://www.paho.org/per/images/st ories/FtPage/2013/PlanENSOPCRD-6julio2013_1v.pdf?ua=1

Organización Mundial de la Salud $\left(\begin{array}{llll}2 & 0 & 1 & 7\end{array}\right)$. O b t e $\mathrm{n} \mathrm{i} \mathrm{d} \mathrm{o} \mathrm{:}$ http://www.who.int/mediacentre/f actsheets/fs282/es/
RPP Noticias (2016). Dispositivos electrónicos pueden provocar estrés $\mathrm{v}$ i s u a 1. O b t e n i d o d e ht t p;//rpp.pe/vida-yestilo/salud/dispositivoselectronicos-pueden-provocarestrés-visual-noticia-946416. 